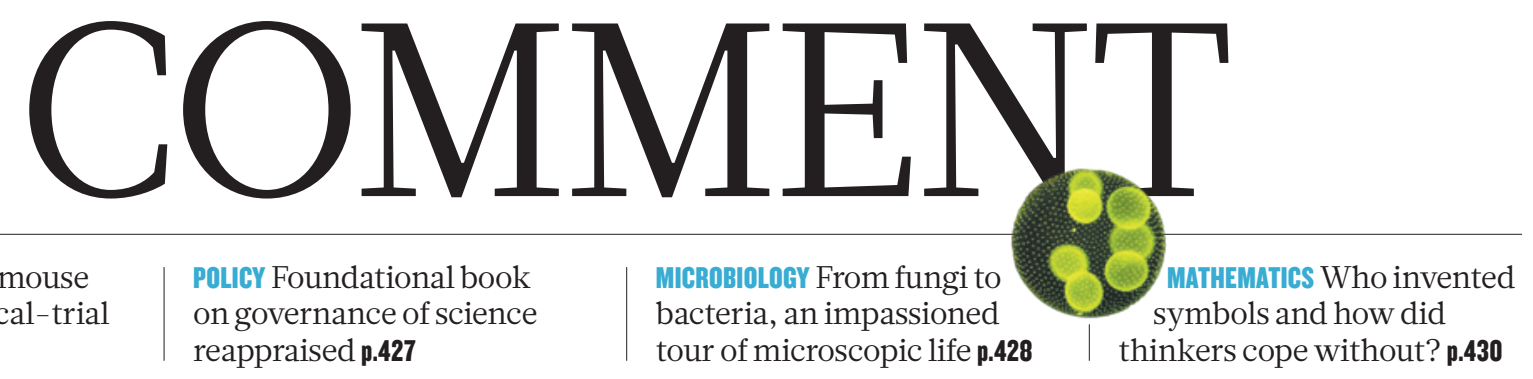

TRANSLATION Improve mouse studies to save clinical-trial resources $\mathbf{p . 4 2 3}$ tional book reappraised $\mathbf{p . 4 2 7}$

\section{michobiotocr From fungito} tour of microscopic life $\mathbf{p . 4 2 8}$
MATHEMATICS Who invented hinkers cope without? p.430

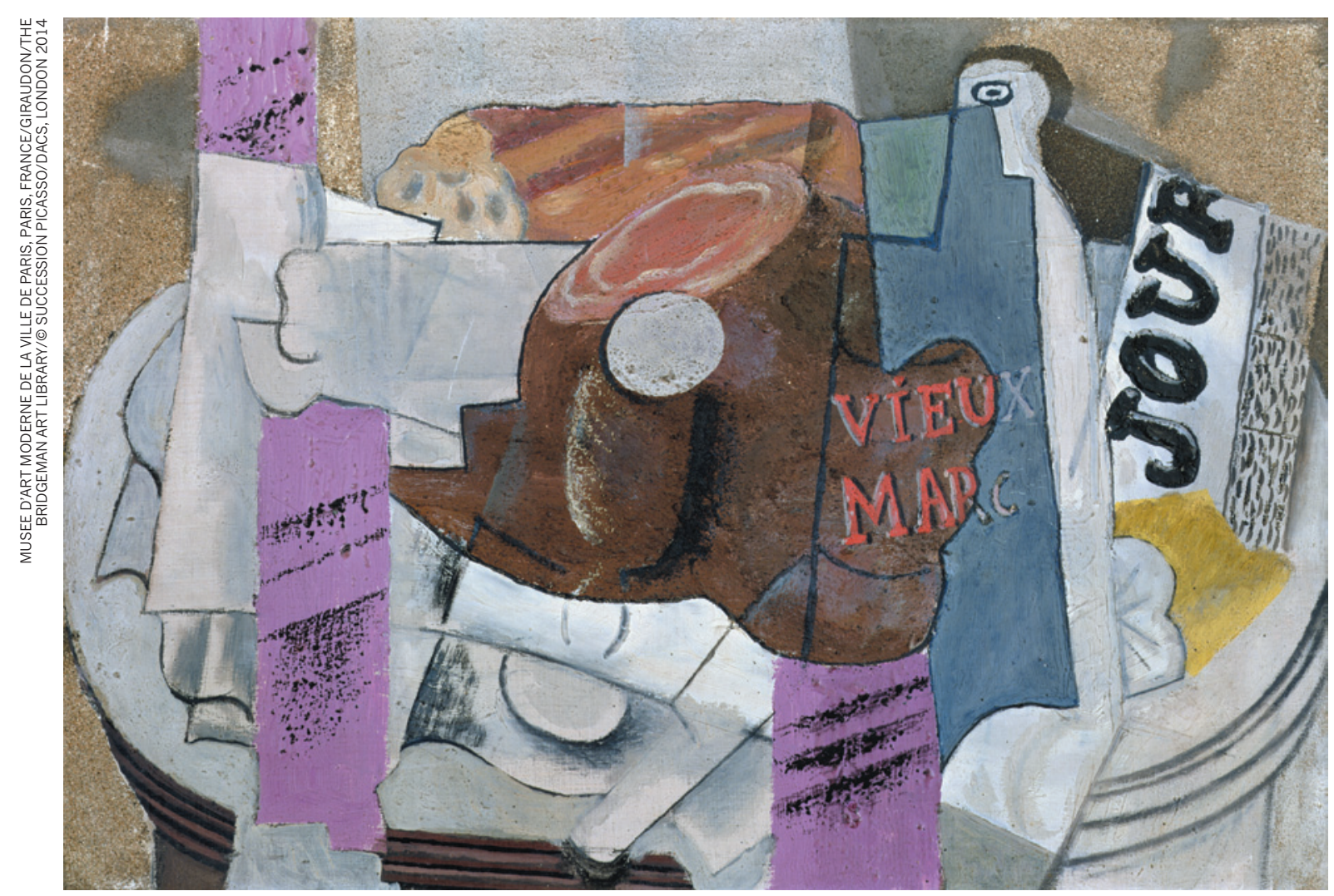

Pablo Picasso, Le Vieux Marc (oil on canvas), 1912.

\title{
QBism puts the scientist back into science
}

\section{A participatory view of science resolves quantum paradoxes and finds room in classical physics for 'the Now', says N. David Mermin.}

$\mathrm{P}$ hysical science describes the objective external world: particles, waves and fields; how they change in time; and how they give rise to the forms of matter, terrestrial and extraterrestrial, microscopic and macroscopic. This world makes itself known to each of us through our own private internal perceptions. Yet physical science has ignored the 'subject' - the scientist - even though their subjective experience constitutes their only link with the external world.

In Nature and the Greeks ${ }^{1}$, Austrian physicist Erwin Schrödinger traced the removal of the subject from science back more than two millennia. Alongside the spectacular success of physical science, this exclusion of personal experience has given rise to some vexing and persistent puzzles and paradoxes.

Two such unrelated long-standing problems are both resolved by recognizing that the perceiving subject has as important a role to play in understanding the nature of physical science as does the perceived object.

The first problem is the notorious disagreement, confusion and murkiness that for almost a century has plagued the foundations of quantum mechanics, in spite of the theory's extraordinary usefulness and power. The second, less famous, problem has been with us at least as long: there seems to be nothing $>$ 
> in physics that singles out 'the present moment'. Albert Einstein called this the problem of 'the Now'. Both problems are symptoms of the exclusion from physical science of the perceiving subject, and are solved by restoring what the ancient Greeks removed.

\section{QUANTUM MECHANICS}

Schrödinger wrote in a little-known 1931 letter $^{2}$ to German physicist Arnold Sommerfeld that quantum mechanics "deals only with the object-subject relation". Another founder of quantum mechanics, Danish physicist Niels Bohr, insisted in a 1929 essay $^{3}$ that the purpose of science was not to reveal "the real essence of the phenomena" but only to find "relations between the manifold aspects of our experience".

In spite of these early hints, it was only in the twenty-first century that US physicist Christopher Fuchs and British-German physicist Rüdiger Schack ${ }^{4-6}$ put forth an understanding of quantum mechanics that restored the balance between subject and object. They call their new point of view 'QBism': $\mathrm{Q}$ is for quantum and $\mathrm{B}$ is for Bayesian - a view of probability that includes an agent who makes bets and updates odds. QBism attributes the muddle at the foundations of quantum mechanics to our unacknowledged removal of the scientist from the science.

Much of this muddle is associated with the 'wavefunction' that quantum mechanics assigns to a physical system. This irritatingly uninformative term reveals the lack of clarity present in the field from its very beginning in 1925. People argue to this day about whether wavefunctions are real entities, like stones or ripples on a pond, or mathematical abstractions that help us to organize our thinking, like the calculus of probabilities.

Fuchs and Schack adopt the latter view. They take a wavefunction to be associated with a physical system by an agent - me, for example, based on my past experience. I use the wavefunction, following rules laid down by quantum mechanics, to calculate the likelihood of what I might experience next, should I choose to probe further. Depending on what I then perceive, I can update the wavefunction on the basis of that experience, allowing me to better assess my subsequent expectations.

People who believe wavefunctions to be as real as stones have invested much effort in searching for objective physical mechanisms responsible for such changes in the wavefunction: a novel manifestation of gravity, for example, or a new kind of fundamental allpervasive friction. But according to QBism, the change is only in my personal expectations, which I revise to accommodate my new experience.

Another celebrated part of the muddle produced by the exclusion of the perceiving subject is 'quantum non-locality', the belief of

some quantum physicists and many mystics, parapsychologists and journalists that an action in one region of space can instantly alter the real state of affairs in a faraway region. Thousands of papers have been written about this mysterious action at a distance over the past 50 years. A clue that the only change is in the expectations of the perceiving subject ${ }^{7}$ is that to learn anything about such alterations one must consult somebody in the region where the action took place.

Most physicists who have paid some attention to QBism have rejected this explicit intro-

"The Now is neither an illusion nor a spurious manifestation of temporal chauvinism." duction of subjective personal experience into science, together with its consequences for our understanding of quantum physics. It offends their sense that science is strictly objective.

QBists are often charged with solipsism: a belief that the world exists only in the mind of a single agent. This is wrong. Although I cannot enter your mind to experience your own private perceptions, you can affect my perceptions through language. When I converse with you or read your books and articles in Nature, I plausibly conclude that you are a perceiving being rather like myself, and infer features of your experience. This is how we can arrive at a common understanding of our external worlds, in spite of the privacy of our individual experiences.

\section{THE NOW}

The QBist conversation can be broadened to include issues in which neither quantum mechanics nor probability plays a part, such as the problem of the Now, which arises in purely classical (pre-quantum) physics. I change the term to 'CBism' when describing applications of the QBist view of science in such classical settings. Here $\mathrm{C}$ stands for classical and B, for Bohr, whose wisdom went beyond quantum mechanics when he taught that physical science studies our experience.

Philosopher Rudolph Carnap ${ }^{8}$ recalls that the problem of the Now worried Einstein seriously. Einstein told him that the experience of the present moment means something special for mankind, essentially different from the past and the future, and that physics cannot describe such a difference. Carnap described Einstein as painfully resigned to the inability of science to grasp this experience.

The issue for Einstein was not the famous revelation of relativity that whether or not two events in two different places happen at the same time can depend on your frame of reference. It was simply that physics seems to offer no way to identify the Now even at a single event in a single place, although a local present moment - Now - is evident to each and every one of us as undeniably real. How can there be no place in physics for something as obvious as that?

My Now - my current state of affairs - is a special event for me while it is happening. I can tell my Now from earlier events, which I only remember, and from later events which I can only anticipate or imagine. The status of an event as my Now is transitory: it becomes a memory as subsequent Nows emerge.

Yet clear, evident and banal as this is to us all, there is no Now in the usual physical description of space and time. Physicists represent all the events experienced by a single person as a line in four-dimensional space-time, called that person's 'world-line'. There is nothing about any point on my world-line that singles it out as my Now.

When I recently mentioned to an eminent theoretical physicist that I was writing an essay explaining how the QBist view of science solves the strictly classical problem of the Now, he said: "Ah, you're going to explain why we all have that illusion." And a distinguished philosopher of science recently derided the attitude that there ought to be a Now on my world-line as "chauvinism of the present moment" .

But the Now is neither an illusion nor a spurious manifestation of temporal chauvinism. The problem of the Now is laid to rest by recognizing the mistake behind the conclusion that it is missing from our physical description of the world. That is the very error that led us into the quantum muddle: the exclusion of personal experience from physical science. Einstein's pain at the inability of science to contain a Now was of a piece with his stubborn refusal to accept quantum mechanics as an adequate view of the world.

Physicists reify space-time. They elevate it from a four-dimensional diagram used to record their experience into the kind of "real essence" that Bohr warned us not to seek. My space-time diagram lets me represent events from past experiences, along with deductions or conjectures about events that were not experienced or have yet to happen. By identifying my abstract diagram with an objective reality, I fool myself into regarding that diagram as a four-dimensional arena in which my life is lived. Actual experiences are spread out in time and in space, and actual clocks used to associate times with our experiences are extended physical objects. To represent the rich spatio-temporal structure of human experience as mathematical points in a space-time continuum is a smart strategic simplification, but we ought not to confuse our actual experience with a cartoon.

That there is a place for the present moment in physics becomes obvious when I take my experience of it as the reality it clearly is to me and recognize that space-time is an abstraction that I construct to organize such 
experiences. At any moment I can represent my past experience as my world-line, terminating in my Now. As it turns into a memory, I expand my diagram to contain my subsequent Nows. The motion of my Now along my world-line reflects the fact that as my watch advances I acquire more experiences to record.

This provides the place in physics for the Now of any one person. But could the problem of the Now lie in relating the present moments of several different people? When you and I are communicating face-to-face I cannot imagine that a live encounter for me could be only a memory for you, or vice versa. When two people are together at an event, if the event is Now for one of them, then it is Now for both. Although this is only an inference for each person, I take it to be as fundamental a feature of two perceiving subjects as the Now is for a single subject.

Our present moments must overlap at every one of our meetings - whenever we have a conversation, move apart and then come back together and have another conversation. But throughout human history people have only moved at low speeds. The complicating effect of relativistic 'time dilation' - the slowing down of rapidly moving clocks - on the advances of our different individual Nows has been far too small to notice. We can, however, entertain the question of whether our present moments would coincide when we came back together, regardless of how rapidly we moved back and forth and regardless of how long the journey.

It is a basic fact of relativity that my personal time - the progress of my present moment - keeps pace with the reading of my watch. If it did not, I would be aware that the rate of my watch had changed as it moved with me, in violation of Einstein's (and Galileo's) principle of relativity. This is all we need. Consider two twins. When they are together at home, their Nows coincide. Then Alice flies off to a nearby star at $80 \%$ of the speed of light, turns around and flies back home to Bob at the same speed. Relativity requires that if Bob's watch has advanced ten years in the meantime, Alice's has advanced only six. But because each of their present moments has advanced in step with the watch each is carrying, the moment of their reunion continues to be Now for them both.

So it is incorrect to claim that physics has nothing to say about local Nows at single events. Physics predicts that our experiences of the Now will continue to have the same familiar features in a future world of interstellar travel at speeds near the speed of light, even for the distinct Nows of many different agents.

Because it solves diverse conundrums in quantum mechanics as well as in the strictly classical problem of the Now, QBist (or CBist) thinking needs to be taken more seriously by physicists. It is time to consider what other foundational puzzles can be resolved by restoring the balance between subject and object in physical science.

As another Viennese investigator even more famous than Schrödinger - Sigmund Freud - put it in 1927 (ref. 10): "The problem of a world constitution that takes no account of the mental apparatus by which we perceive it is an empty abstraction."

N. David Mermin is emeritus professor in the Laboratory of Atomic and Solid State Physics, Cornell University, Ithaca, New York, USA. He started to take QBism seriously while at the Stellenbosch Institute for Advanced Study, South Africa. e-mail:david.mermin@cornell.edu

1. Schrödinger, E. Nature and the Greeks and Science and Humanism (Cambridge Univ. Press, 1996).

2. Schrödinger, E. Eine Entdeckung von ganz ausserordentlicher Tragweite (ed. von Meyenn, K.) 490 (Springer, 2011).

3. Bohr, N. Atomic Theory and the Description of Nature 18 (Cambridge Univ. Press, 1934).

4. Caves, C. M., Fuchs, C. A. \& Schack, R. Phys. Rev. A 65, 022305 (2002).

5. Fuchs, C. A. Preprint at http://arxiv.org/ abs/1003.5209 (2010).

6. Fuchs, C. A. \& Schack, R. Rev. Mod. Phys. $\mathbf{8 5}$, 1693-1715 (2013)

7. Fuchs, C. A., Mermin, N. D. \& Schack, R. Preprint at http://arxiv.org/abs/1311.5253 (2013).

8. Carnap, R. The Philosophy of Rudolf Carnap (ed. Schilpp, P. A.) 37-38 (Open Court Publishing, 1963)

9. Price, H. Science 341, 960-961 (2013).

10. Freud, S. The Future of an Illusion, in Mass Psychology and Other Writings (Penguin, 2004).

\section{Make mouse studies work}

\section{More investment to characterize animal models can boost the ability of preclinical work to predict drug effects in humans, says Steve Perrin.}

$\mathrm{M}$ ranslational research. Even after animal studies suggest that a treatment will be safe and effective, more than $80 \%$ of potential therapeutics fail when tested in people. Animal models of disease are frequently condemned as poor predictors of whether an experimental drug can become an effective treatment. Often, though, the real reason is that the preclinical experiments were not rigorously designed $^{1,2}$.

The series of clinical trials for a potential therapy can cost hundreds of millions of dollars. The human costs are even greater: patients with progressive terminal illnesses may have just one shot at an unproven but promising treatment. Clinical trials typically

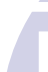

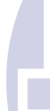

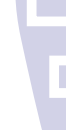

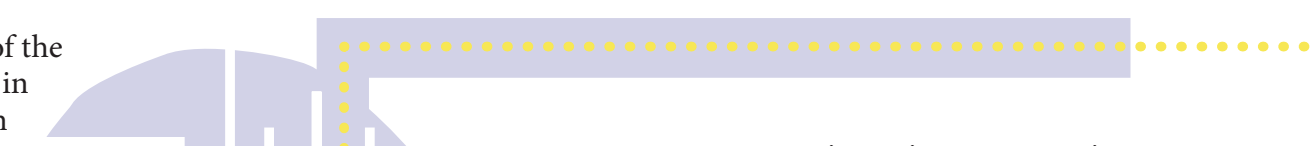

